\title{
Behindertenrecht und Behindertenpolitik in der Kommune aus kommunaler Sicht
}

\section{Helmut Kneppe}

$\begin{array}{ll}\text { I. Einleitung } & 141\end{array}$

1. Sozialraum 141

2. Personenzentrierung 142

3. Aufgabenverständnis der Kommunen 142

II. Kommentierung aus kommunaler Sicht 143

1. Ausgangspunkte der Ausführungen 143

2. Die Realisierung - aus dem Blickwinkel eines kommunalen Praktikers $\quad 144$

III. Fazit

\section{Einleitung}

Der Kommentar erfasst folgende Aspekte: Unter dem Eindruck der bisher vorgetragenen Tagungsbeiträge insgesamt werde ich in aller Kürze auf drei Stichworte eingehen. Diese Stichworte spreche ich aus meinem Blickwinkel ganz praktischer Planungsund Umsetzungsbelange im Sozialraum an. Hierbei bitte ich um ihr Verständnis für die eben wegen der Kürze an der Oberfläche gebliebene Argumentation:

\section{Sozialraum}

Aus meiner von praktischen Zusammenhängen geprägten Sicht ist der Sozialraum für den betroffenen Menschen (Adressaten einer sozialen Leistung) in der Regel keine universell und über bestimmte Koordinaten objektiv zu beschreibende absolute Fläche bzw. kein absoluter Raum. Trotzdem ist ein Sozialraum natürlich räumlich zu verstehen und hat insoweit auch die Aufgabe, räumliche Bezüge und Zusammenhänge herzustellen. Er ist in seiner konkreten Gestaltung immer abhängig von objektiven und subjektiven Rahmenbedingungen des einzelnen (behinderten) Menschen und seinem individuellen (Lebens-) Umfeld. 
Wie auch immer sich Gemeinschaften, Gesellschaften oder auch Staaten organisiert haben, die sozialen Belange der Menschen, ihrer Familien und auch der Gemeinschaften selbst sind nach meiner Kenntnis von der Organisationsebene wahrgenommen worden, die ihnen am nächsten war. Das Grundprinzip dieser örtlichen Kompetenzzuweisung, der Beauftragung der örtlichen Ebene nah am Menschen, haben wir in unsere heutige Situation übernommen:

Der Sozialraum ist der Ursprung und das Urgeschäft der Kommunen.

Im Sozialraum findet der Mensch die Wertschätzung, Kompetenzen, Strukturen, Leistungen, Angebote, Menschen und Partner, um sein Leben nach seinen Möglichkeiten unbehindert und selbstbestimmt führen und am Leben in seinem Sozialraum teilhaben zu können. Der Sozialraum ist sein gelingendes Lebensumfeld „nah am Menschen“.

Die in und für dieses Lebensumfeld zuständigen Akteure gelingender Teilhabe arbeiten in diesem Sinne vernetzt und koordiniert miteinander. Ihnen gelingt es, bedarfsgerecht Infrastruktur-, Geschäftsmodellentwicklung und Hilfeplanung im Einzelfall zu verbinden.

\section{Personenzentrierung}

Personenzentrierung verstehe ich als eine wichtige Perspektive für die Anforderungen an die Gestaltung gelingender Sozialleistungen. Personenzentriert interpretiere ich dementsprechend i.S. der Kundenperspektive der Balanced-Score-Card für soziale Organisationen (herausgearbeitet für not for profit Organisationen ${ }^{1}$ ).

Das heißt, im Rahmen der gesetzlich geregelten Ansprüche und Möglichkeiten sind der (behinderte) Mensch und seine Perspektive (Bedarfe, Lebensentwurf) die inhaltlich gestaltende Bezugsebene. Es sind daher konsequent und dem Grunde nach konsistent auch die Budgets auf die Person und/oder auf den Sozialraum bezogen zu gestalten.

Hieraus folgt: Nicht der Sozialraum oder der/die Sozialplaner/innen stehen im Mittelpunkt, sondern der (behinderte) Mensch und seine Bedarfe im Kontext sozialräumlicher Belange. Ich grenze „personenzentriert“ in diesem Sinne inhaltlich zu den Begriffen „struktur- und prozesszentriert“, „kompetenzzentriert“ sowie „ressourcen- und finanzzentriert" ab.

\section{Aufgabenverständnis der Kommunen}

Die erfolgreiche Steuerung des Ressourceneinsatzes bei knappen Ressourcen kann nur gelingen, wenn die Kommunen schon zu einem Zeitpunkt die Zugänge in soziale Leistungen aktiv gestalten, zu dem wir aufgrund der gesetzlichen Regelungen noch

1 Stollmann, BSC für soziale Organisation, Regensburg 2008. 
nicht zuständig sind. Unser Aufgabenverständnis muss/müsste also konsequent proaktiv gestaltend und nicht lediglich verwaltend sein.

Zum Beleg, dass rechtliche Grundlagen für ein solches Aufgabenverständnis an einigen Stellen schon heute vorhanden sind, darf ich zustimmend beispielsweise auf die Darstellungen durch Felix Welti und Gerhard Igl ${ }^{2}$ Bezug nehmen.

Eine solchermaßen aktiv gestaltende Aufgabenwahrnehmung ist in der Praxis jedoch offensichtlich nicht konsistent zu haushaltsrechtlichen Regelungen und Entscheidungen. Finanzaufsicht stuft mit dem Ziel der Haushaltskonsolidierung die Wahrnehmung derart präventiv angelegter Maßnahmen als „freiwillige Aufgabe“ ein und gestattet diese im Kontext einer Haushaltssicherung oder eines Nothaushaltes in der Regel nicht.

Zahlreiche gute Ansätze zur Wahrnehmung einer modernen Sozialplanung und Steuerungsunterstützung stoßen in einer solchen Lage an die Grenzen des tatsächlich Machbaren.

Aus meiner Sicht müssen hier auch wir Kommunen uns bewegen und verstärkt den tatsächlichen Willen und Mut aufbringen, aktiv zu gestalten. Wir dürfen bei aller berechtigten Anmahnung bedarfsgerechter Ressourcen uns nicht auf eine Opferrolle beschränken und eine entsprechende Mentalität entwickeln.

\section{Kommentierung aus kommunaler Sicht}

\section{Ausgangspunkte der Ausführungen}

a) Soziale Rechte, Ansprüche und Leistungen haben in der Regel die Aufgabe, ökonomische und/oder nicht ökonomische Beratungs-, Assistenz- und Hilfebedarfe der betroffenen Menschen sowie auch ihrer Familien bzw. persönlichem Umfeld zu befriedigen bzw. Beiträge dazu zu leisten.

Sozialpolitisches Wirkungsziel der zu diesem Zweck begründeten Rechte, Ansprüche und Leistungen ist es, die eigenständige und selbstverantwortliche Lebensführung und Teilhabe der Betroffenen zu fördern bzw. diese zu ermöglichen.

b) Zur Erreichung dieser Ziele werden auf Gesetzesebene und unterhalb dieser Anspruchsvoraussetzungen, (angemessene) Beratungs-, Assistenz- und Hilfeleistungen sowie der berechtigte Personenkreis abstrakt beschrieben und in Form von Ansprüchen und Rechten (z.B.) als persönliche -, Sach- oder Geldleistung artikuliert und für die Berechtigten mehr oder weniger formgebunden verfügbar gemacht.

Die Realisierung obliegt sehr vielfältig aufgestellten und positionierten Sozialleistungsträgern. Ihre Leistungserbringung ist in der Regel nicht im wünschenswerten Maße vernetzt.

2 Siehe deren Beiträge, S. 87 und S. 119 in diesem Band. 
Den Kommunen (kreisfreie Städte, Kreise, kreisangehörige Städte und Gemeinden, kommunale Verbände) kommt in diesem Gesamtsystem aus dem Blickwinkel der Leistungsberechtigten strukturell, prozessbezogen und inhaltlich eine wichtige Position zu. Die betroffenen Menschen weisen ihnen eine hohe Lösungs- und Handlungskompetenz $\mathrm{zu}$.

c) Unsere Sozialleistungssysteme stehen unter einem enormen Druck. Die Systeme selbst und deren Planungs-, Aufgaben- und Kostenträger werden zunehmend an ihre Leistungsgrenzen und auch darüber hinaus geführt. Schon jetzt bzw. in absehbarer Zeit werden die verfügbaren Ressourcen, Kompetenzen und Möglichkeiten überfordert sein. Es sei denn, wesentliche Rahmenbedingungen des „Gelingens sozialer Leistungserbringung“" werden mit nachhaltiger Wirkung verändert.

Wir müssen daher schon heute das Leistungs-, Organisations- und Finanzierungssystem im $\mathrm{Ob}$ und im Wie gründlich hinterfragen!

d) Aus meinem Blickwinkel des Praktikers führt die oben beschriebene Gefahr der Überforderung der Systeme zunehmend zu Reaktionen auf allen Ebenen.

Auf der kommunalen Ebene reichen diese von der Etablierung aktiv gestaltender präventiv wirkender Konzepte bis hin zu absolut destruktiven Entscheidungen im Rahmen der Haushaltskonsolidierung, die Auswirkungen bis auf die Leistungsebene im Einzelfall haben können. Ganz entscheidende Bedeutung haben hierbei die wirtschaftliche Leistungsfähigkeit der Kommunen und die darauf folgenden Reaktionen.

Destruktive und aus der Sicht der Betroffenen sogar kontraproduktiv wirkende Einzelentscheidungen müssen konstruktiv in Frage gestellt bzw. beendet werden.

Hieraus folgt: Wir brauchen auf der strategischen Entscheidungs-, Planungs- und Steuerungsebene und der operativen Leistungsebene die Entwicklung neuer strategischer und ganzheitlich angelegter Handlungskonzepte. Ich denke, hier liegt ein wichtiges Aufgabenfeld für die Sozialleistungsträger, insbesondere auch die Kommunen, sowie Wissenschaft und Forschung. Der von Wissenschaft und Forschung sowie manchen Sozialleistungsträgern schon seit einigen Jahren eingeschlagene Weg der Entwicklung aktiver Planungs- und Steuerungskonzepte - aus meiner Sicht ganz bewusst auch in Anlehnung an den „betriebswirtschaftlichen Methodenkoffer“ - sollte intensiviert werden.

\section{Die Realisierung - aus dem Blickwinkel eines kommunalen Praktikers}

a) Die Erreichung der gewollten Ziele/Wirkung gelingt, wenn die Leistungen

- „nah am Menschen“ und

- tatsächlich, verlässlich, qualitätsvoll, wertschätzend und finanziert sind.

„Nah am Menschen“ heißt: bedarfsgerecht; ortsnah; vernetzt und eingebunden; finanziert. „Nah am Menschen“ heißt daher zwingend auch: Soziale Leistungserbringung 
muss personenzentriert und ganzheitlich gestaltet sein. Personenzentriert und ganzheitlich gestaltet bedeutet aus der Sicht der betroffenen Menschen und der Sozialleistungsträger:

- Die Infrastruktur, das Leistungsangebot, die Geschäftsmodelle müssen dem entsprechend entwickelt und für die betroffenen Menschen im Sozialraum verfügbar sein (Stichwort: Care-Management);

- die Leistung, der Leistungsprozess kommen passend im jeweiligen Quartier an und entfalten die gewünschte Wirkung (Stichwort: Case-Management);

- die für eine wertschätzende Leistungserbringung notwendigen Kompetenzen und Ressourcen sind im erforderlichen notwendigen Maße verlässlich und bezahlbar vorhanden.

Das Gelingen sozialer Leistungen entsprechend diesen Maßgaben zu organisieren, bedarf einer diesen Anforderungen entsprechenden Sozialplanung, Sozialraumplanung sowie Organisation und Steuerung der Leistungserbringung, also einer planvollen, zielgerichteten, partnerschaftlichen und disziplinübergreifenden Interaktion aller an der sozialen Leistungserbringung beteiligten Akteure.

b) Die Ebene, auf der alle beteiligten Akteure, die notwendigen Kompetenzen und die betroffenen Menschen zusammenkommen bzw. vertreten sind, ist die Kommune.

Nur auf kommunaler Ebene, bei und mit den Menschen vor Ort, können soziale Leistungen ihre gewollte Wirkung in vollem Umfang entfalten. Letztlich werden dort Ansprüche in konkrete Leistungen übersetzt. Nur auf der kommunalen Ebene haben soziale Leistungen einen persönlichen Ansprechpartner und sind nicht anonym. (Stichwort „Dienstleistung aus einer Hand“). Hierbei ist es ein Qualitätsmerkmal und nicht Hindernis, dass die kommunale Ebene Unterebenen aufweist und in dieser Republik sehr unterschiedlich gestaltet ist. Unsere vielfältigen kommunalen Strukturen sind letztlich auch Garanten für die Nähe zum Menschen. Die Kommunen sind die „geborenen“ (und legitimierten) Gestalter ihrer Sozialräume und Partner ihrer Menschen, Familien und regionalen Akteure. Der Sozialraum ist das Urgeschäft der Kommunen.

c) Aus der bisherigen - ganz bewusst nicht juristisch, sondern sozialpolitisch aus der Sicht der betroffenen Menschen vorgenommenen - Ableitung folgt zwangsläufig, dass sich damit auch eine entsprechende Verpflichtung und Aufgabenstellung der kommunalen Akteure ergibt. Unter Aufgreifen des von Gerhard $\operatorname{Igl}^{3}$ dargestellten Gedankens darf auch aus meiner Sicht eine „Entpflichtung“ bzw. „Selbstentpflichtung“ der Kommunen nicht Raum greifen.

Ich sehe im Rahmen der aktuellen Inklusionsdiskussion die Entwicklung, dass wir Kommunen uns formal hinter den noch ausstehenden Regelungen des Bundes und der Länder sowie unseren Zuständigkeiten verstecken. Findet auch hier wieder eine Selbstentpflichtung statt?

3 S. 119 in diesem Band. 
Jedoch darf es auch für Bund und Land keine Selbstentpflichtung zu Lasten der Kommunen geben. Bund und Land überfordern ihre kommunalen Partner, indem sie kommunale Leistungen beauftragen und die erforderlichen Mittel nicht, nicht ausreichend bzw. nur ,ausreichend“ im Sinne einer recht eigenen Sichtweise/Bedarfsermittlung zur Verfügung stellen.

d) Was ist vor Ort notwendig, damit soziale Leistungserbringung gelingt? Die nachfolgenden Beispiele beziehen sich auf Klarheit und Wertschätzung in Aufgabe, Auftrag, Zielen und Wirkungen, Zielgruppe, Verantwortlichkeiten, Terminen und Ressourcen:

- Benennung der Aufgabe;

- aus welchem Grund und Anlass greifen wir diese auf;

- welche Wirkungen und Ziele verfolgen wir?;

- für wen, Zielgruppe?;

- welche Ist-Situation wollen wir in welche Soll-Situation entwickeln?;

- mit welchen Maßnahmen, Projekten, Produkten erreichen wir die Ziele, die Wirkungen?;

- wer ist verantwortlich und kümmert sich um Planung, Produktion, Evaluation und Steuerung?;

- welche Partner und Beteiligten sind einzubinden?;

- womit wird die Aufgabe, der Auftrag erledigt, was ist erforderlich? Das bezieht sich auf Personal (Kompetenzen), Sach-, Betriebs- und Hilfsmittel, IT TUIV, sowie die Finanzen.

Diese Punkte sind abgeleitet aus verschiedenen Steuerungsansätzen und bedürfen inhaltlich und methodisch der Konkretisierung. Einer Anforderung die sich zuvorderst an den Aufgabenverantwortlichen wendet und diese zur Schaffung leistungsfähiger Angebote verpflichtet.

Insbesondere die Bereitstellung der für die Aufgabenwahrnehmung notwendigen Ressourcen ist in der Praxis häufig mangelhaft ausgeprägt. Für mich steckt darin auch ein Signal mangelnder Wertschätzung und mangelnden Respekts gegenüber Betroffenen und Handelnden.

e) Eine ehrliche Analyse der allgemeinen und örtlichen Ausgangssituation muss die folgenden Rahmenbedingungen vor Ort erfassen:

- Demografische Entwicklung, heruntergebrochen auf die örtliche Situation;

- Knappheit der verfügbaren Ressourcen, soziale Leistungen im Wettbewerb um kommunale Ressourcen;

- Aufgaben- und Methodenkritik, Kosten-Nutzen-Analysen;

- neues kommunales Finanzmanagement NkF, Kaufmännische Buchführung, Doppik, Verwaltungsunternehmen, unternehmerische Führung;

- Aufgabenbezogene Bestandsaufnahme, Ist-Erhebung, Repräsentativbefragungen. 
Der politische und fachliche Gestaltungswille der Kommune muss von den kommunalen Gremien beschlossen, kommuniziert und verlässlich gelebt werden - als politische Kommune und als Sozialleistungsträger.

f) In der örtlichen Situation ist Ganzheitlichkeit und gemeinsame Zielorientierung in ländlichen Gebieten methodisch anders herzustellen als in städtischen Gebieten. Dies liegt nicht nur an den Anforderungen der Versorgung in der Fläche. Das liegt auch am „Kirchturmdenken“.

In den Städten, Gemeinden und Kreisen, Regiestellen und Servicestellen Soziales sollen örtliche „Kümmerer“ etabliert werden. Sie sollen aus den Rathäusern und Kreishäusern heraus mit den betroffenen Menschen, den Aufgaben- und Kostenträgern, den Dienstleistern und dem bürgerschaftlichen Engagement gemeinsam passgenaue Infrastruktur, Strategien und Angebote entwickeln.

\section{Fazit}

Gelingende Sozialpolitik und gelingende Sozialleistung zu gewährleisten und dies auch noch zur Zufriedenheit der Leistungsempfänger, ihres Umfeldes und der Aufgaben- und Kostenträger ist eine „Übung für Fortgeschrittene“.

Grundvoraussetzungen des Gelingens sind

- die Verankerung der Leistungserbringung im Sozialraum

- und dem entsprechend die Akzeptanz und positive gestaltende Annahme dieser Aufgabe durch die Kommunen.

Die schon bestehenden gesetzlichen und auch untergesetzlichen Regelungen, sich dieser Aufgabe im erforderlichen Maße legitimiert anzunehmen, sind aus meiner kommunalen Sicht vielfältig. Sie werden derzeit durch uns Kommunen sehr unterschiedlich aufgegriffen. In einer Gesamtsicht nutzen wir diese Handlungsfelder und die in ihnen liegenden Gestaltungsräume wohl noch nicht umfassend.

Ich sehe hier wichtige Handlungsfelder und Entwicklungsmöglichkeiten für uns Kommunen sowie Gestaltungsraum für den Gesetz- und Verordnungsgeber, Planung, Koordination und Vernetzung personenzentrierter Hilfeleistung im örtlichen Sozialraum kommunal verlässlich zu verankern.

Im Sinne einer Zielbeschreibung bedeutet dies soziale Leistungserbringung in modernen Dienstleistungsunternehmen zu organisieren, zu steuern und sicherzustellen.

Die Kommunen sind jedoch aus meiner Perspektive für die Wahrnehmung der ihnen obliegenden Aufgaben in der geforderten Qualität insgesamt unterfinanziert.

Es dürfte nicht zulässig sein, ihnen Aufgaben zu übertragen, bestehende Aufgaben zu erweitern, ohne die Aufgabenfinanzierung verlässlich zu regeln. Alternativ wäre die geforderte Produktqualität anzupassen. 
Wir Kommunen haben hierbei auch weiterhin unseren Beitrag der eigenen Strukturund Prozessoptimierung zu erbringen.

Die Wahrnehmung, Übertragung bzw. Erweiterung von Aufgaben (z.B. als Sozialleistungsträger) muss getragen sein von einer eindeutigen Auftragslage, Klarheit in der Zielsetzung, in den Zuständigkeiten und in der Ausstattung mit Ressourcen.

Eine der Gesetzesentscheidung vorgelagerte gründliche und umfassende Produkt- bzw. Leistungsplanung, Ziel- und Maßnahmenabstimmung sowie auch Planung der notwendigen Transfermittel und einzusetzenden Ressourcen sollte eine wichtigere Position einnehmen.

Sozialleistung gelingt, wenn sie personenzentriert gestaltet, im Sozialraum verankert ist, nah am Menschen ankommt sowie verlässlich, qualitativ hochwertig, wertschätzend und finanziert erbracht wird. Aus der praktischen Sicht ergibt sich zum heutigen Thema folgende Ableitung:

Für mich und für die Kommunikation in meinen kommunalen Bezügen nehme ich aus dieser Fachtagung ein hohes Maß, ja sogar die Aufforderung mit, den aktiv gestaltenden Ansatz unserer kommunalen Sozialpolitik weiter zu verfolgen, sogar noch zu verstärken.

Alter und Behinderung stehen in den Zusammenhängen dieser Fachtagung für mich beispielhaft. Sie können - wenn auch nicht beliebig - so doch durch andere Beschreibungen von Lebenslagen ersetzt werden. Daraus folgt, dass meine Ausführungen eine auch über das Thema hinausgehende Dimension haben.

Wenn sich das Sozialleistungsrecht in drei Dimensionen (Schutz bei Pflegebedürftigkeit, medizinische Rehabilitationsfähigkeit, spezielle Gesundheitsleistungen) abbildet, so resultiert daraus, dass das Sozialleistungsrecht das Entstehen des zugeordneten Leistungsanspruches an eine defizitäre Lebenssituation bindet.

Der auslösende Zustand muss die Teilhabe, die Lebensqualität des Betroffenen beeinträchtigen. Sozialleistung repariert demnach und gestaltet nur eingeschränkt.

Sozialpolitisch muss aus alledem folgend sehr viel stärker als dies heute schon möglich ist und geschieht Präventionsleistung vor Eintritt des Leistungsanspruches verankert werden. Besonders gilt dies aus meiner Sicht für Sozialleistungen, die nicht beitragsfinanziert sind wie z.B. die Jugendhilfe, die Sozialhilfe, die Behindertenhilfe, die Wohnungshilfe.

Ziel sollte sein, den Eintritt des Leistungsfalles zu verhindern bzw. mindestens zu verzögern, d.h., den Zugang, den Verbleib und das Verlassen in/von Sozialleistungen aktiv zu steuern.

Nur auf der kommunalen Ebene im örtlichen Sozialraum laufen all diese Stränge zusammen. Es bedarf einer ganzheitlich angelegten Planung, Vernetzung und Koordination der örtlichen Akteure. Ansprüche, Bedarfe, Strukturen und Prozesse müssen zusammengeführt werden.

In der aktuellen Ausgangslage ist es wesentlich an den Kommunen, diesen Handlungs- 
bedarf aufzugreifen und weiter zu entwickeln, und zwar über Zuständigkeits- und Aufgabengrenzen hinweg.

Auch aus der kommunalen Sicht teile ich den in dieser Fachtagung an zahlreichen Stellen herausgearbeiteten und dargestellten Handlungsbedarf, gelingende Teilhabe im Sozialraum aktiv aufzugreifen und zu gestalten. Dies bedarf einer verstärkten und gemeinsamen Anstrengung aller Beteiligten, aller Akteure im Sozialraum. 
2. Kinder und Jugendliche mit Behinderungen 